\title{
Anionic Long-Circulating Quantum Dots for Long-Term Intravital Vascular Imaging
}

\author{
Haolu Wang ${ }^{1}$, Haotian Yang ${ }^{1}{ }^{(}$, Zhi Ping $X u^{2}{ }^{\circledR}, X$ Xin Liu ${ }^{1}$, Michael S. Roberts ${ }^{1,3}$ and \\ Xiaowen Liang ${ }^{1, *}$ \\ 1 Therapeutics Research Group, The University of Queensland Diamantina Institute, \\ The University of Queensland, Translational Research Institute, Brisbane, QLD 4102, Australia; \\ h.wang21@uq.edu.au (H.W.); haotian.yang@uq.net.au (H.Y.); xin.liu@uq.edu.au (X.L.); \\ m.roberts@uq.edu.au (M.S.R.) \\ 2 Australian Institute for Bioengineering and Nanotechnology, The University of Queensland, St Lucia, \\ Brisbane, QLD 4072, Australia; gordonxu@uq.edu.au \\ 3 School of Pharmacy and Medical Science, University of South Australia, Adelaide, SA 5001, Australia \\ * Correspondence: x.liang@uq.edu.au; Tel.: +61-7-3443-7487
}

Received: 17 October 2018; Accepted: 17 November 2018; Published: 20 November 2018

\begin{abstract}
A major impediment to the long-term in vivo vascular imaging is a lack of suitable probes and contrast agents. Our developed mercaptosuccinic acid (MSA) capped cadmium telluride/cadmium sulfide (CdTe/CdS) ultrasmall quantum dots (QDs) have high fluorescent quantum yield, long fluorescence lifetime and long half-life in blood, allowing high resolution long-term intravital vascular imaging. In this study, we showed that these QDs can be used to visualize the in vivo the vasculature in normal and cancerous livers in mice using multiphoton microscopy (MPM) coupled with fluorescence lifetime imaging (FLIM), with cellular resolution $(\sim 1 \mu \mathrm{m})$ up to $36 \mathrm{~h}$ after intravenous injection. Compared to highly regulated and controlled sinusoids in normal liver tissue, disordered, tortuous, and immature neovessels were observed in tumors. The utilized imaging methods have great potential as emerging tools in diagnosis and monitoring of treatment response in cancer.
\end{abstract}

Keywords: quantum dots; blood vessels; intravital imaging

\section{Introduction}

Optical-based intravital imaging of vasculature is an emerging modality for studying vascular structure, function, and angiogenesis. Changes in the number and spacing of vessels, permeability of the vasculature and vascular function have been implicated in many diseases, including malformation and cancer [1]. Although many probes and contrast agents have been developed for imaging of microvasculature, issues related to stability and bioavailability have yet to be overcome $[1,2]$. A multivalent and biologically compatible platform for the development of fluorescent imaging agents is still needed for long-term in vivo imaging. As a biological imaging agent, quantum dots (QDs) possess a number of distinct advantages over other nanoparticles (NPs) including high quantum yield, enhanced photostability, narrow emission band, and long fluorescence lifetime [3-5]. QDs applied for in vivo imaging of tumor vasculature were firstly reported by Cai et al. [6], where the majority of QDs were found in the tumor vasculature with few binding to tumor cells. Smith et al. also reported the binding events of QDs to the tumor vasculature using intravital microscopy [7]. This study confirmed that QDs did not extravasate but only targeted the vascular integrin $\alpha \mathrm{V} \beta 3$, which is involved in tumor angiogenesis. However, these studies only reported in vivo QDs distribution in tumors at the organ-level or by ex vivo sections. The limited resolution and penetration depth of conventional 
in vivo imaging techniques make it difficult to obtain a clear real-time dynamics of QDs at the cellular level in vivo.

We have successfully synthesized long-circulating QDs in our laboratory and their in vivo fate has been fully investigated [4,5,8]. Our results showed these QDs mainly distributed in the liver and kidney. We then further investigated the spatiotemporal disposition of QDs in the liver and kidney by multiphoton microscopy (MPM) [4]. These QDs were evenly distributed in the blood vessels of these organs and circulated for a long time, but were not taken up by hepatocytes and tubular cells [4]. Here, we further applied these ultrasmall water-dispersible cadmium telluride/cadmium sulfide $(\mathrm{CdTe} / \mathrm{CdS}) \mathrm{QDs}$ for the long-term intravital imaging of vasculature in normal and cancerous livers.

\section{Materials and Methods}

\subsection{Chemicals and Cells}

Ilium xylazile and ketamine hydrochloride were purchased from Bayer Australia (Pymble NSW, Australia). Hepa 1-6 cells were obtained from ATCC (Manassas, VA, USA) and maintained in vitro under cell culture conditions recommended by ATCC.

QDs were mercaptosuccinic acid (MSA, Sigma-Aldrich, MO, USA) capped cadmium telluride/cadmium sulfide (CdTe/CdS, Sigma-Aldrich) particles, recently developed in our laboratory [4]. These QDs have an average particle diameter of $3.5 \mathrm{~nm}$ determined by transmission electron microscopy (JEOL1010, JEOL, Tokyo, Japan). The hydrodynamic diameter of these QDs was measured to be $4.2 \mathrm{~nm}$. They display abroad excitation $(<500 \mathrm{~nm})$ with an emission peak of about $630 \mathrm{~nm}$. The zeta potential measured was $-37 \mathrm{mV}$ [4].

\subsection{Animal Models}

All animal procedures were approved by the health sciences ethics committee of the University of Queensland (ethics number is 521/12 and approved on the year of 2013) and were carried out in accordance with the legislation of Australian authorities for the care and use of experimental animals.

Male 8-week-old BALB/c nude mice purchased from the Animal Resource Centre (Perth, Western Australia) were used in this study. Hepatocellular carcinoma was induced by intrahepatic implantation of 10 ${ }^{7}$ Hepa1- 6 cells into BALB/c nude mice via open surgical technique and imaging procedures were performed after 14 days [9]. Three mice per time point were randomly selected for time course studies. Each mouse was injected via the tail vein with $145.5 \mathrm{pmol} / \mathrm{g}$ of MSA-QDs (particle concentration was about $14.55 \mathrm{nmol} / \mathrm{mL}$ ). The injection volume was $0.01 \mathrm{~mL} / \mathrm{g}$. Control mice were injected by the same volume of PBS. Serial sacrifices were carried out at $5 \mathrm{~min}, 1 \mathrm{~h}, 4 \mathrm{~h}, 8 \mathrm{~h}, 24 \mathrm{~h}, 7$ days, and 30 days following dosing of MSA-QDs. Plasma samples were collected at each time point and were analyzed for quantification of QDs on the basis of Cd contents. Pharmacokinetic parameters were determined by compartmental analysis, and all the data were fitted by a two-compartment model.

\subsection{Quantification of the QD Concentration in Plasma}

QD concentration in mouse plasma was based on the determination of cadmium concentration by inductively coupled plasma-mass spectrometry (ICP-MS, Agilent 7700, Agilent Technologies, Tokyo, Japan). Briefly, plasma were collected, dried, weighted, and digested with $70 \%$ nitric acid on a hot plate at $100{ }^{\circ} \mathrm{C}$. The $\mathrm{Cd}^{2+}$ concentration of these digested samples was measured using calibration curve ranged from 0 to $500 \mathrm{ng} / \mathrm{mL}$ [3].

\subsection{MPM-FLIM}

Short and long-term intravital images were collected from $30 \mathrm{~s}$ after injection using our published protocols [8,10]. MPM was performed using the DermaInspect system (Jen-Lab GmbH, Jena, Germany) equipped with an ultrashort ( $85 \mathrm{fs}$ pulse width) pulsed mode-locked 80-MHz titanium sapphire laser (MaiTai, Spectra Physics, Mount View, CA, USA). The excitation wavelength was set to $740 \mathrm{~nm}$ for liver 
autofluorescence and $900 \mathrm{~nm}$ for QDs signals, with an emission signal range of 350 to $650 \mathrm{~nm}$ established through the use of a BG39 bandpass filter (BG39, Schott glass color filter, Schott MG, Mainz, Germany). Images were recorded with oil-immersion $40 \times$ objectives (Carl Zeiss, Germany). The laser power was set to $20 \mathrm{~mW}$ for $10 \times$ magnification imaging, and the acquisition time for obtaining the images was $7.4 \mathrm{~s}$ per frame. Each image was $179 \times 179 \mu \mathrm{m}$ wide at a resolution of $512 \times 512$ pixels. For mouse blood imaging, blood was collected after mouse scarification and was placed on the slide for imaging.

For fluorescence lifetime imaging (FLIM), a time-correlated single-photon counting (TCSPC) SPC-830 detector (Becker \& Hickl, Berlin, Germany) was incorporated into the MPM system. The TCSPC module constructs a photon distribution across the $\mathrm{x}$ and $\mathrm{y}$ coordinates of the scan area. Each FLIM scan was performed using an exposure of $7.4 \mathrm{~s}$ at an acquisition image size of $214 \times 214 \mu \mathrm{m}$. Fluorescence emission was spectrally resolved between linearly arranged photon counters through the use of dichroic filters in the beam path. The emission light was collected spectrally in a channel of 350 to $450 \mathrm{~nm}$ at the excitation of $740 \mathrm{~nm}$ for nicotinamide adenine dinucleotide (NADH), while the channel of 515 to $620 \mathrm{~nm}$ at the excitation of $740 \mathrm{~nm}$ for QD fluorescent signal.

\subsection{Histological Analysis}

Liver, kidney and spleen tissues were collected at day 7 or 30 after QDs intravenous injection in mice. These samples were fixed in formalin, then embedded in paraffin, sectioned, and stained with hematoxylin and eosin $(\mathrm{H} \& \mathrm{E})$. Then the stained slides were examined by light microscopy.

\subsection{Data Analysis}

Quantitative analysis of the fluorescence intensity images was processed using ImageJ $1.44 \mathrm{p}$ (National Institutes of Health, Bethesda, MD, USA).

FLIM images were analyzed using SPCImage software 4.9.7 (Becker \& Hickl, Berlin, Germany). Lifetime distributions for $\mathrm{NADH}$ were obtained by fitting photon count $\mathrm{F}(\mathrm{t})$ profiles from each image to a bi-exponential decay function $\left(\mathrm{F}(\mathrm{t})=\alpha_{1} \mathrm{e}-\mathrm{t} / \tau_{1}+\alpha_{2} \mathrm{e}-\mathrm{t} / \tau_{2}\right)$. Two lifetimes, $\tau_{1}$ and $\tau_{2}$ represent the fast and slow decay lifetimes of free and protein-bound NADH, respectively. The amplitudes $\alpha_{1}$ and $\alpha_{2}$ represent the relative concentration fraction of nicotinamide adenine dinucleotide (NADH), where $\alpha_{1}+\alpha_{2}=1$ [11]. $\tau_{m}$ is the weighted average lifetime calculated from $\tau_{1}$ and $\tau_{2}$ and their relative amplitudes $\left(\tau_{\mathrm{m}}=\alpha_{1} \tau_{1}+\alpha_{2} \tau_{2}\right)$.

\section{Results}

These anionic ultrasmall QDs with the size of $3.5 \mathrm{~nm}$ in diameter that were mainly distributed in the blood vessels, and cannot extravasate, be taken up by parenchymal cells in organs or excreted into urine and feces up to 30 days [4]. Only a small amount of these particles were found to be taken up only by phagocytic cells (Kupffer cells and endothelial cells in liver, and mesangial cells in kidney) after injection $[5,8]$. In particular, our previous study has found that these QDs were gradually taken up by mesangial cells up to 30 days post-injection, which was detected by TEM imaging [5]. This result further supports QDs were not excreted through the body up to 30 days. We evaluated the QD concentrations in plasma by ICP-MS and they displayed a bi-exponential decay (Figure 1) in systemic blood circulation with long half-lives of $7.67 \mathrm{~h}\left(\mathrm{t}_{1 / 2 \alpha}\right)$ and $2363.19 \mathrm{~h}\left(\mathrm{t}_{1 / 2 \beta}\right)$ (Table 1$)$. Hence, these QDs circulated in the body for a long time and are suitable for the long-term intravital imaging of vasculature.

Table 1. Pharmacokinetic parameters of quantum dots (QDs) after intravenous injection fitted by a two-compartment model.

\begin{tabular}{cccc}
\hline Parameter (Unit) & Value & Parameter (Unit) & Value \\
\hline CL $(\mathrm{mL} / \mathrm{h})$ & 0.006 & $\mathrm{k}_{12}\left(\mathrm{~h}^{-1}\right)$ & 0.075 \\
Vc $(\mathrm{mL})$ & 3.11 & $\mathrm{k}_{21}\left(\mathrm{~h}^{-1}\right)$ & 0.014 \\
CLd $(\mathrm{mL} / \mathrm{h})$ & 0.23 & $\mathrm{t}_{1 / 2 \alpha}(\mathrm{h})$ & 7.67 \\
Vss $(\mathrm{mL})$ & 20.38 & $\mathrm{t}_{1 / 2 \beta}(\mathrm{h})$ & 2363.19 \\
\hline
\end{tabular}




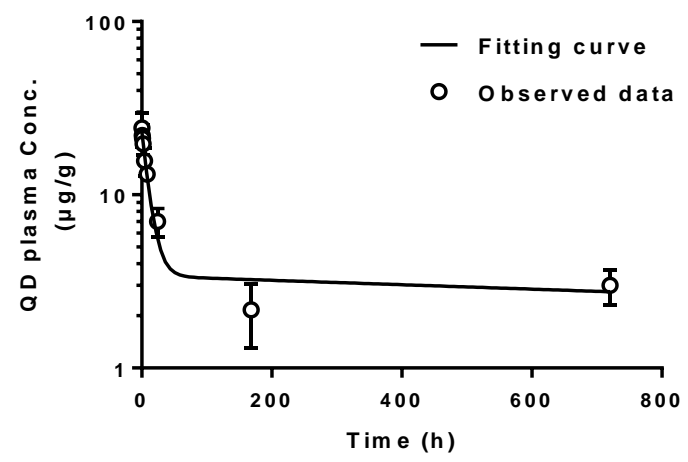

Figure 1. Plasma concentration of QDs following an intravenous injection detected by ICP-MS and it was fitted by a two-compartment model. Symbols represent the mean experimental data and error bars represent standard deviation. The solid lines are simulated by a two-compartment model.

We further performed in vitro experiments using MPM coupled with FLIM to determine the fluorescence lifetimes of mouse blood after QDs administration (Figure 2). The average weighted lifetime $\left(\tau_{\mathrm{m}}\right)$ was determined to be about $9 \mathrm{~ns}$ in mouse blood and $10 \mathrm{~ns}$ in plasma after QDs administration. These results indicated that the fluorescent propertis of these QDs were stable and they have longer lifetimes compared to normal tissue autofluorescence (2-3 ns) [3].

Liver images were obtained at the excitation wavelength of $900 \mathrm{~nm}$ to selectively capture QD fluorescent signal since liver autofluorescence of NADH is absent at this excitation wavelength [3]. As shown in Figure 3B,F, vasculatures of normal liver tissue and hepatocellular carcinoma were clearly visualized by highly intense fluorescence signals of QDs after $30 \mathrm{~min}$ of intravenous injection. Unlike highly regulated and controlled sinusoids in normal liver tissues, tumor tissues exhibited disordered and tortuous vasculature, mainly due to tumor aniogenesis and lack of blood perfusion. Neovessels were found to be connected with larger vessels. These images further confirmed that QDs were retained in the vasculature and were not taken up by hepatocytes or tumor cells (Figure 3A,B,E,F). As shown in Figure 4, the fluorescence of QDs reached maximum at $2 \mathrm{~min}$ post-injection, and was evenly distributed in blood vessels. The QDs labeled vascular images were obvious up to $36 \mathrm{~h}$ after injection, but QD signals gradually disappeared at $48 \mathrm{~h}$ post-injection.
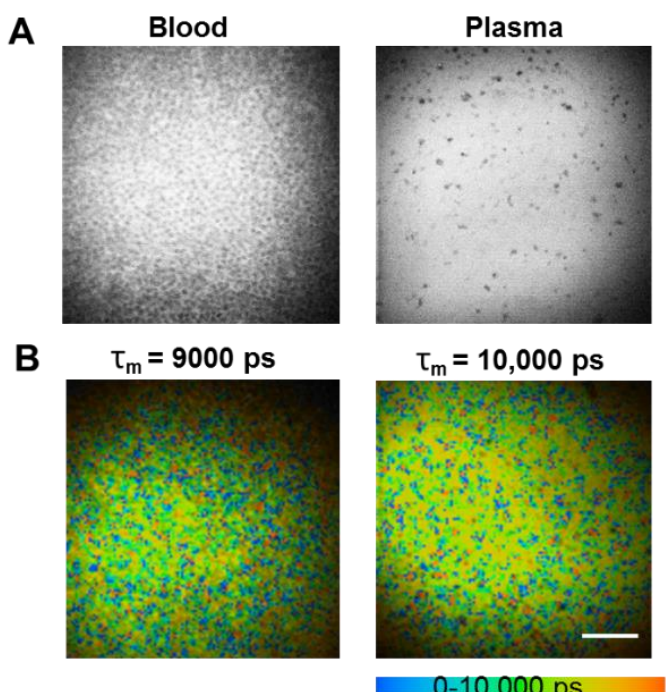

Figure 2. Fluorescence intensity image of mouse blood after QDs administration recorded at $\lambda_{\mathrm{Exc}} / \lambda_{\mathrm{Em}}$ : 900/515 to $620 \mathrm{~nm}$ (A); Fluorescence lifetime properties of mouse blood after QDs administration measured by MPM-FLIM (B). Pseudocolored fluorescence lifetime image ( $\tau_{\mathrm{m}}$ : 0-10,000 ps; blue-green-red) recorded at $\lambda_{\mathrm{Exc}} / \lambda_{\mathrm{Em}}: 900 / 515$ to $620 \mathrm{~nm}$ (scale bar: $160 \mu \mathrm{m}$ ). 

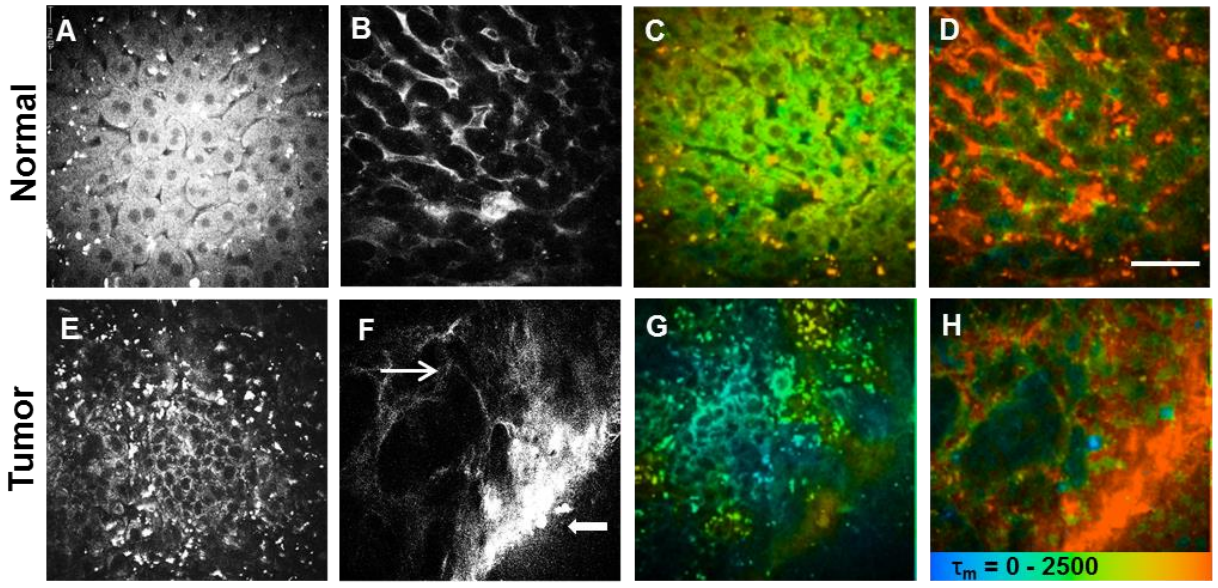

Figure 3. Multiphoton microscopy coupled with fluorescence lifetime imaging (MPM-FLIM) images of normal liver tissue and hepatocellular carcinoma after QDs injection. Narrow arrow indicates disordered and tortuous vasculature of hepatocellular carcinoma with inefficient blood perfusion and filled arrow indicates larger vessels connected to the tumor vasculature. (A,E) Fluorescence intensity image recorded at $\lambda_{\mathrm{Exc}} / \lambda_{\mathrm{Em}}: 740 / 350$ to $450 \mathrm{~nm} ;(\mathbf{B}, \mathbf{F})$ Fluorescence intensity image recorded at $\lambda_{\mathrm{Exc}} / \lambda_{\mathrm{Em}}$ : $900 / 515$ to $620 \mathrm{~nm}$; (C,G) Pseudocolored fluorescence lifetime image $\left(\tau_{\mathrm{m}}: 0-2500 \mathrm{ps}\right.$; blue-green-red) recorded at $\lambda_{\mathrm{Exc}} / \lambda_{\mathrm{Em}}: 740 / 350$ to $450 \mathrm{~nm}$ or $(\mathbf{D}, \mathbf{H})$ recorded at $\lambda_{\mathrm{Exc}} / \lambda_{\mathrm{Em}}: 900 / 515$ to $620 \mathrm{~nm}$. (Scale bar: $40 \mu \mathrm{m}$ ).

A
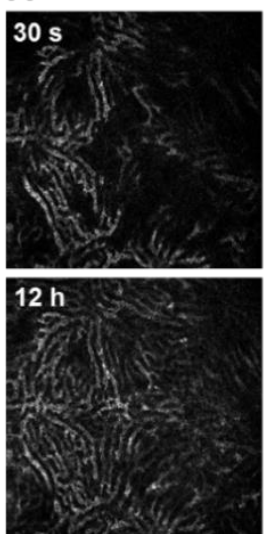
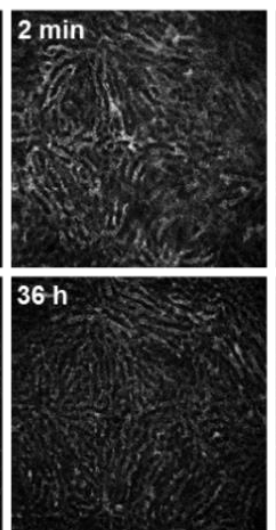

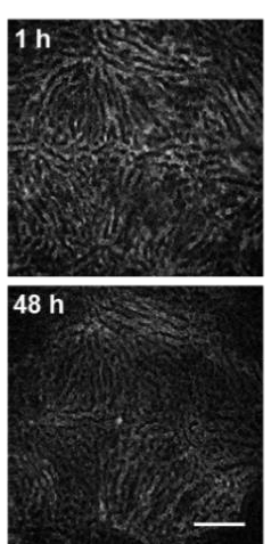

B

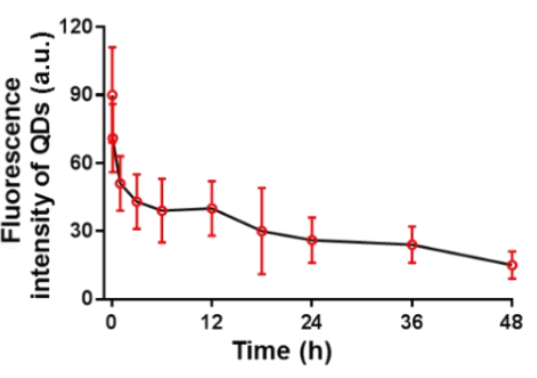

Figure 4. Time profile of QDs intensity in vasculature of normal liver after bolus injection. (A) Real-time hepatic disposition of QDs, (Scale bar: $160 \mu \mathrm{m}$ ); (B) Time profile of QDs intensity per pixel. The symbols represent measured data and the line represents the connecting curve. Error bar represents the standard deviation $(n=3)$.

In addition to fluorescent signals, FLIM could map the spatial distribution of fluorophores based on fluorescence lifetime, the average time of an electron stays in the excited state before returning to the ground state [11]. Lifetime changes also can be used to differentiate fluorophores from biological environments and also detect environmental changes, such as protein binding or $\mathrm{pH}$ [10]. These anionic ultrasmall QDs are particularly suitable for FLIM because their fluorescence lifetime (typically $\geq 10 \mathrm{~ns}$ ) is much longer than those of organ autofluorescence (2-3 ns) [3], which also have been confirmed in Figure 2. As shown in Figure 3D,H, FLIM images further clearly confirmed the QDs labeled vasculatures of normal liver tissue and hepatocellular carcinoma, where orange color represents longer lifetime of QDs (Figure 3D,H) and green color (Figure 3C,G) represents shorter lifetimes of autofluorescence from hepatocytes or tumor cells.

Finally, we did the histological analysis of key organs of mice treated by QDs, which allows for detailed microscopic evaluation and histological assessment of tissue interactions. Since QDs are 
mainly accumulated in the liver, spleen, and kidney for a long period, it is necessary to understand their toxicity in these organs. As shown in Figure 5, there were no apparent histopathological abnormalities or lesions observed in these organs exposed with QDs. Hepatocytes were observed to be normal and there were no signs of inflammatory response in QDs treated tissues. The glomerular structure of the kidney was clear and no pathological changes were observed in the spleen. H\&E staining images of such organs showed the same properties as those of control tissues, with no signs of overt degeneration, inflammation and necrosis in any of examined tissues. The lower toxicity of these QDs to the organs could be attributed to the formation of CdS shell on the core CdTe of QDs [12].

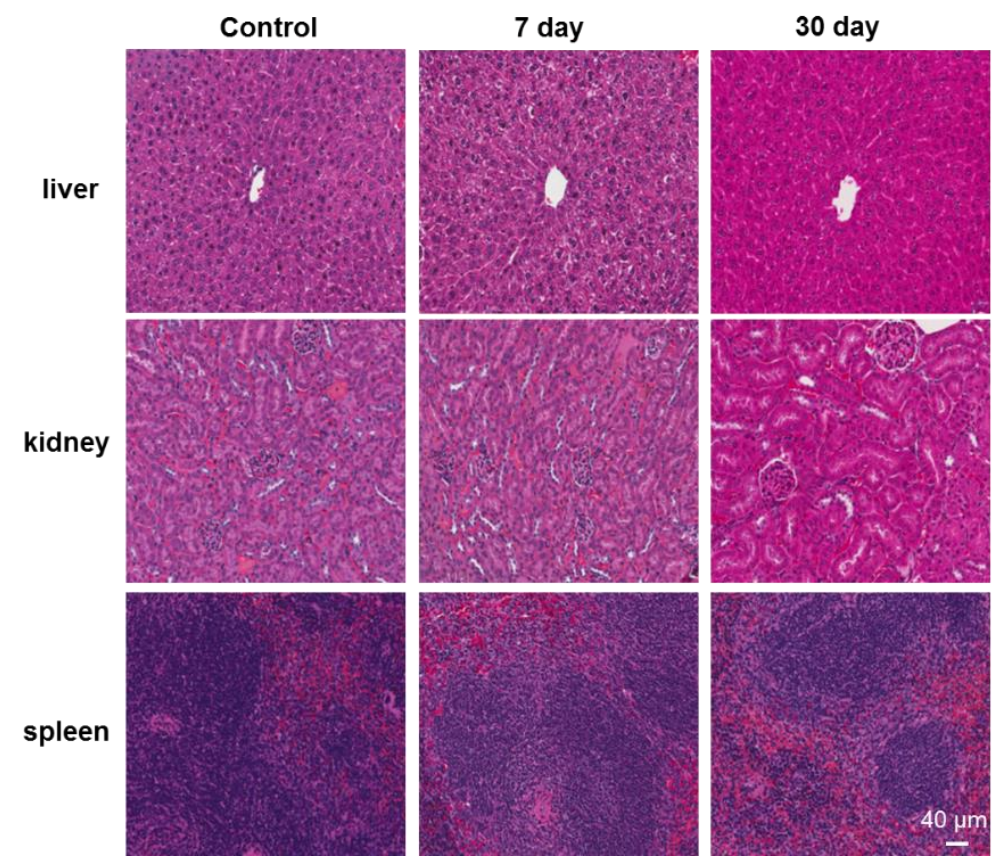

Figure 5. Representative organ histology images of control and QDs treated mice. QDs treated organs were collected 7 days or 30 days after intravenous injection of QDs. (Scale bar: $40 \mu \mathrm{m}$ ).

\section{Discussion}

In this study, we found that the anionic ultrasmall QDs present new opportunities for intravital vascular imaging as they retain in vascular for a long time after systematic injection without uptake by parenchymal cells. Compared to conventional in vivo imaging, MPM has the advantages of less photobleaching and photodamage, and has considerably enhanced imaging penetration depth due to less scattered multiphoton excitation in samples [10]. Fresh, unprocessed and unstained organs can be imaged in vivo or ex vivo by MPM [13]. Most vascular contrast agents are transient imaging reagents with imaging time windows ranging from a few minutes to several hours, depending on the properties of the probe. In contrast, ultrasmall inorganic NPs have longer half-lives in the body in comparison with larger NPs, organic fluorescent dyes or bovine serum albumin (BSA) and transferrin conjugated vasculature agents [4]. We have previously reported that cationic QDs of similar size can be readily excreted into urine, while anionic ultrasmall QDs have prolonged blood circulation and high photostability [3], which allows repeated and enhanced angiogenesis imaging. Compared to conventional technique for quantifying blood flow, MPM has been opening up a new avenue for simultaneous imaging depiction of the cellular and extracellular microstructures in tissues [14]. Here, we reported the pharmacokinetics of these QDs, and demonstrated that their fluorescence remained in vasculature up to $36 \mathrm{~h}$ after injection under MPM, allowing long-term intravital imaging. These QDs have advantages over organic nanoparticles (e.g., polymers and liposomes) for tumor angiogenesis imaging since they have less extravasation in tumor due to the rigid cores. However, most of these published or commercialized QDs will readily leak from the capillaries within $6 \mathrm{~h}$ [15]. It is of interest 
to note that our non-targeted QDs show little or no extravasation from the vasculature up to $36 \mathrm{~h}$ post introduction into the tumor xenograft bearing mice. These NPs can be used separately, or in combination with other proteins/antibodies to the in vivo imaging system.

Vascular imaging is of particular importance in tumor biology where imaging can be used to study the growth and progression of cancer, and assess the efficacy of anti-tumor and anti-angiogenesis drugs [16]. Once the diameter of a tumor reaches 1 to $2 \mathrm{~mm}$, diffusion of nutrients and oxygen from surrounding host vasculature becomes insufficient, leading to angiogenesis, the formation of new blood vessels from preexisting vasculature [17]. Tumor-induced angiogenesis is connected to the host's circulatory system, and plays a key role in cancer invasion and metastasis. Therefore, vasculature of cancer could be used as diagnostic and prognostic markers and the excellent targets for chemotherapy [17]. Since the angiogenesis of tumors highly reached the surface [18], imaging of the vasculature near the tumor surface is feasible for detecting angiogenesis using MPM-FLIM [19].

In summary, we show that anionic ultrasmall QDs can be used to visualize the in vivo vasculature in normal and cancerous livers in mice, with cellular resolution $(\sim 1 \mu \mathrm{m})$, up to $36 \mathrm{~h}$ after intravenous injection. MPM coupled with FLIM has great potential as emerging tools in diagnosis and monitoring of treatment response in liver cancer.

Author Contributions: Conceptualization, H.W. and X.L. (Xiaowen Liang); methodology, H.W. and Z.P.X.; validation, H.Y. and X.L. (Xiaowen Liang); investigation, X.L. (Xin Liu); writing-original draft preparation, H.W., X.L. (Xiaowen Liang), and H.Y.; writing-review and editing, X.L. (Xiaowen Liang), X.L. (Xin Liu), Z.P.X. and M.S.R.; supervision, M.S.R. and X.L. (Xiaowen Liang); funding acquisition, X.L. (Xiaowen Liang) and M.S.R.

Funding: The study was supported by grants from National Health and Medical Research Council of Australia (APP1125794) and UQ Development Fellowship.

Conflicts of Interest: The authors declare no conflict of interest. The funders had no role in the design of the study; in the collection, analyses, or interpretation of data; in the writing of the manuscript, or in the decision to publish the results.

\section{References}

1. Lewis, J.D.; Destito, G.; Zijlstra, A.; Gonzalez, M.J.; Quigley, J.P.; Manchester, M.; Stuhlmann, H. Viral nanoparticles as tools for intravital vascular imaging. Nat. Med. 2006, 12, 354-360. [CrossRef] [PubMed]

2. Hilderbrand, S.A.; Weissleder, R. Near-infrared fluorescence: Application to in vivo molecular imaging. Curr. Opin. Chem. Biol. 2010, 14, 71-79. [CrossRef] [PubMed]

3. Liang, X.; Grice, J.E.; Zhu, Y.; Liu, D.; Sanchez, W.Y.; Li, Z.; Crawford, D.H.; Le Couteur, D.G.; Cogger, V.C.; Liu, X.; et al. Intravital Multiphoton Imaging of the Selective Uptake of Water-Dispersible Quantum Dots into Sinusoidal Liver Cells. Small 2014. [CrossRef] [PubMed]

4. Liang, X.W.; Wang, H.L.; Grice, J.E.; Li, L.; Liu, X.; Xu, Z.P.; Roberts, M.S. Physiologically Based Pharmacokinetic Model for Long-Circulating Inorganic Nanoparticles. Nano Lett. 2016, 16, 939-945. [CrossRef] [PubMed]

5. Liang, X.W.; Wang, H.L.; Zhu, Y.; Zhang, R.; Cogger, V.C.; Liu, X.; Xu, Z.P.; Grice, J.E.; Roberts, M.S. Short- and Long-Term Tracking of Anionic Ultrasmall Nanoparticles in Kidney. Acs Nano 2016, 10, 387-395. [CrossRef] [PubMed]

6. Cai, W.B.; Shin, D.W.; Chen, K.; Gheysens, O.; Cao, Q.Z.; Wang, S.X.; Gambhir, S.S.; Chen, X.Y. Peptide-labeled near-infrared quantum dots for imaging tumor vasculature in living subjects. Nano Lett. 2006, 6, 669-676. [CrossRef] [PubMed]

7. Smith, B.R.; Cheng, Z.; De, A.; Koh, A.L.; Sinclair, R.; Gambhir, S.S. Real-time intravital imaging of RGD-quantum dot binding to luminal endothelium in mouse tumor neovasculature. Nano Lett. 2008, 8, 2599-2606. [CrossRef] [PubMed]

8. Wang, H.L.; Liang, X.W.; Mohammed, Y.H.; Thomas, J.A.; Bridle, K.R.; Thorling, C.A.; Grice, J.E.; Xu, Z.P.; Liu, X.; Crawford, D.H.G.; et al. Real-time histology in liver disease using multiphoton microscopy with fluorescence lifetime imaging. Biomed. Opt. Express 2015, 6, 780-792. [CrossRef] [PubMed] 
9. Wang, H.; Thorling, C.A.; Xu, Z.P.; Crawford, D.H.G.; Liang, X.; Liu, X.; Roberts, M.S. Visualization and Modeling of the In Vivo Distribution of Mesenchymal Stem Cells. Curr. Protoc. Stem Cell Biol. 2017, 43, 2B.8.1-2B.8.17. [PubMed]

10. Thorling, C.A.; Liu, X.; Burczynski, F.J.; Fletcher, L.M.; Gobe, G.C.; Roberts, M.S. Multiphoton microscopy can visualize zonal damage and decreased cellular metabolic activity in hepatic ischemia-reperfusion injury in rats. J. Biomed. Opt. 2011, 16. [CrossRef] [PubMed]

11. Lakowicz, J.R.; Szmacinski, H.; Nowaczyk, K.; Berndt, K.W.; Johnson, M. Fluorescence Lifetime Imaging. Anal. Biochem. 1992, 202, 316-330. [CrossRef]

12. Zhu, Y.; Li, Z.; Chen, M.; Cooper, H.M.; Lu, G.Q.; Xu, Z.P. One-pot preparation of highly fluorescent cadmium telluride/cadmium sulfide quantum dots under neutral-pH condition for biological applications. J. Colloid Interf. Sci. 2013, 390, 3-10. [CrossRef] [PubMed]

13. Zipfel, W.R.; Williams, R.M.; Webb, W.W. Nonlinear magic: multiphoton microscopy in the biosciences. Nat. Biotechnol. 2003, 21, 1368-1376. [CrossRef] [PubMed]

14. Wang, B.G.; Konig, K.; Halbhuber, K.J. Two-photon microscopy of deep intravital tissues and its merits in clinical research. J. Microsc-Oxford 2010, 238, 1-20. [CrossRef] [PubMed]

15. Kairdolf, B.A.; Smith, A.M.; Stokes, T.H.; Wang, M.D.; Young, A.N.; Nie, S. Semiconductor quantum dots for bioimaging and biodiagnostic applications. Annu. Rev. Anal. Chem. (Palo Alto Calif.) 2013, 6, 143-162. [CrossRef] [PubMed]

16. Bergers, G.; Benjamin, L.E. Tumorigenesis and the angiogenic switch. Nat. Rev. Cancer 2003, 3, 401-410. [CrossRef] [PubMed]

17. Liu, Z.A.; Peng, R. Inorganic nanomaterials for tumor angiogenesis imaging. Eur. J. Nucl. Med. Mol. I 2010, 37, S147-S163. [CrossRef] [PubMed]

18. Cai, W.B.; Chen, X.Y. Multimodality molecular imaging of tumor angiogenesis. J. Nucl. Med. 2008, 49, 113s-128s. [CrossRef] [PubMed]

19. Jain, R.K.; Munn, L.L.; Fukumura, D. Dissecting tumour pathophysiology using intravital microscopy. Nat. Rev. Cancer 2002, 2, 266-276. [CrossRef] [PubMed] 